\title{
Development of a microfluidic perfusion 3-dimensional cell culture system
}

\author{
D H Park ${ }^{1}$, H J Jeon ${ }^{1}$, M J Kim ${ }^{1}$, X D Nguyen ${ }^{1}, K_{\text {Morten }}^{2}$ and J S Go ${ }^{1 *}$ \\ ${ }^{1}$ School of Mechanical Engineering, Pusan National University, 2, Busandaehak road 63-2, \\ Keumjeong-gu, Busan 609-735, KOREA \\ ${ }^{2}$ Nuffield Department of Obstetrics \& Gynaecology, University of Oxford, Headley Way, \\ Headington, Oxford OX3 9DU, UK \\ E-mail : micros@pusan.ac.kr
}

\begin{abstract}
Recently, 3-dimensional in vitro cell cultures have been gained much attention in biomedical sciences because of their closer relevance between in vitro cell cultures and in vivo environments. This paper presents a microfluidic perfusion 3D cell culture system with consistent control of long-term culture conditions to mimic in vivo microenvironment. It consists of two sudden expansion reservoirs to trap incoming air bubbles, gradient generators to provide a linear concentration, and microchannel mixers. Specifically, the air bubbles disturb a flow in the microfluidic channel resulting in the instability of the perfusion cell culture conditions. For long-term stable operation, the sudden expansion reservoir is designed to trap air bubbles by using buoyancy before they enter the culture system. The performance of the developed microfluidic perfusion 3D cell culture system was examined experimentally and compared with analytical results. Finally, it was applied to test the cytotoxicity of cells infected with Ewing's sarcoma. Cell death was observed for different concentrations of $\mathrm{H}_{2} \mathrm{O}_{2}$. For future work, the developed microfluidic perfusion 3D cell culture system can be used to examine the behavior of cells treated with various drugs and concentrations for high-throughput drug screening.
\end{abstract}

\section{Introduction}

In vitro cell cultures are the most commonly used tool to examine the biological behavior of cells analogous to in vivo microenvironments. Culture conditions such as nutrients and growth factors are controlled to suit the types of target cells. Gases like $\mathrm{CO}_{2}$ and $\mathrm{O}_{2}$ are supplied, and mechanical parameters such as pressure and temperature are also regulated for the culture environment. Generally, these in vitro cell cultures are done in 2-dimensional (hereafter, 2D) well plates. However, the 3-dimensional (hereafter, 3D) spheroids of cancer cells are not observed in the 2D cell culture due to planar proliferation [1-4]. In addition, 2D cell cultures have limitations in examining the effect of diffusion-limited concentration of controlled molecules on cell growth. Additionally, media and nutrients are supplied drop-by-drop into the wells with pipettes, which is certainly different from their continuous 
supply in the in vivo environment. Thus, long term assays using 2D cell cultures under a low concentration condition of media are problematic.

Thus, 3D cell cultures have been attracting much attention due to their potential of achieving a closer relevance between in vitro cell cultures and in vivo environments [2-7]. It is advantageous to maintain wellcontrolled culture conditions consistently during long term cell culture by suppling media continuously with nutrients or growth factors [5, 8, 9]. Carlos E. Caicedo-Carvajal et al. compared a static culture and perfusion culture of U2OS osteosarcoma cells and showed certain differences in cell growth and morphology. Cell proliferation and $\beta$-actin expression were increased in the perfusion 3D cell culture [6,9].

Today, microfluidic perfusion systems have been developed for high-throughput 3D cell culture and dynamic control of flow [5, 7, 8, 10-18]. They have the advantage of a uniform, controlled and continuous perfusion of culture media due to a stable laminar flow in microchannel and high-density integration of various components such as mixers, concentration gradient generators, and filters by using up-to-date microfabrication technology [11]. Kang Tian et al. applied the concentration gradient generator to address different concentrations of molecules in the culture wells for high-throughput analysis and examined optimal chondrocyte differentiation. The optimum concentration of neuroleukin (NLK), which is a multifunctional protein, was newly obtained to be $12.85 \mathrm{ng} / \mathrm{ml}$ for chondrocytes [10].

The microfluidic perfusion 3D cell culture system requires complex channel designs such as multiple channel layers, splitting and recombining channels, and partitioning channels to generate concentration gradients [10, 11, 15-17, 19, 20]. Additionally, during long-term cell culture, the complex microchannel-integrated perfusion 3D cell culture system may suffer from the unwanted introduction of air bubbles [7, 21]. When they are introduced into the system and reside in the channels, the perfusion flow of the media is seriously disturbed, and this affects cell growth [7, 21-26]. Some works have reported the application of micro-filters to prevent air bubbles from entering into the microfluidic perfusion system [7, 21, 23-26]. However, the fabrication of the microfluidic perfusion system becomes more complex and difficult and as a result, high manufacturing is accompanied.

To situate the in-vivo like 3D cell culture properly by using the microfluidic perfusion system, all the components for concentration gradient, mixing, bubble trapping, cell seeding should be integrated, which means that the research on the development of the microfluidic perfusion 3D culture system should be focused on the integrated system rather than on just a specific component of the system. Some papers examined the system consisted of some components such as microchannels to supply medium or drugs continuously [10, 12, 15-18, 20, 27] and cell seeding microchambers structured with micropillars [13, 28, 29] and nano/micro-membranes [30]. However, only few papers have considered the issue of the removal of air bubbles in the integrated microfluidic system. Mostly, they use membrane or external bubble traps to remove air bubbles [13, 14, 20, 29].

In this work, the microfluidic perfusion 3D cell culture system integrated with two sudden expansion reservoirs to trap incoming air bubbles and a linear concentration gradient generator with only two inlets was designed. The culture wells were machined to have the same size with the conventional 2D cell culture wells for user familiarity. It also includes meandered microchannel mixers connected with the linear concentration gradient generator. Its perfusion performances of the trapping of air bubbles, the generation of the linear concentration gradient, and diffusion mixing were evaluated experimentally. Finally, it was used to test the cytotoxicity of cells infected with Ewing's sarcoma at four different concentrations of $\mathrm{H}_{2} \mathrm{O}_{2}$ to demonstrate its feasibility in long term culture assay. 

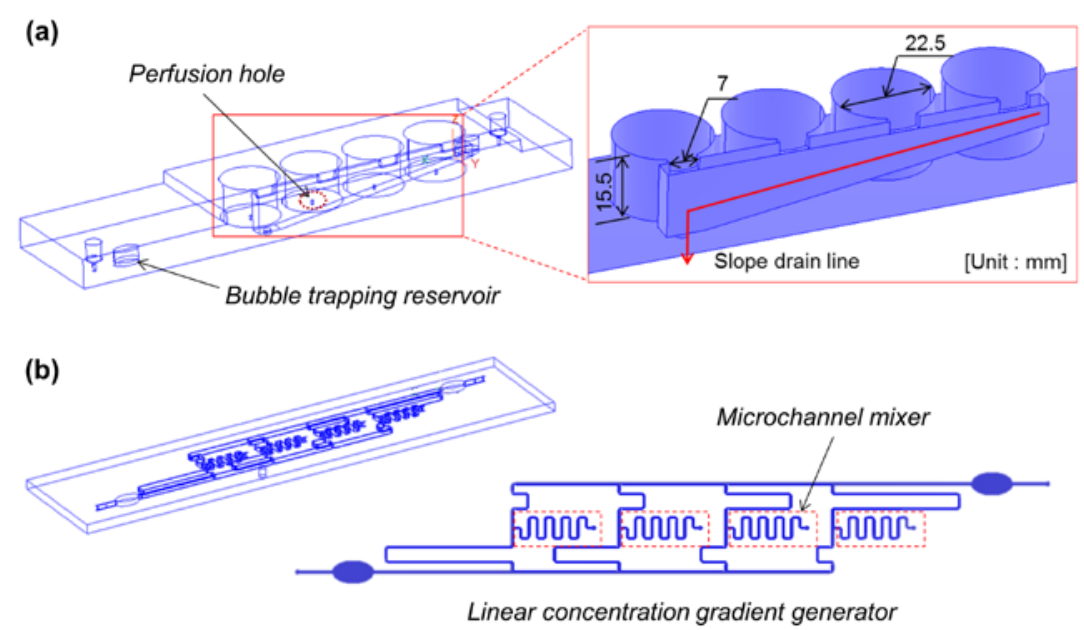

Figure 1. Proposed microfluidic perfusion 3D cell culture system. (a) Upper cell culture well plate, (b) bottom perfusion channel plate.

\section{Design of the microfluidic perfusion 3D cell culture system}

Figure 1 shows the schematic drawing of the proposed microfluidic perfusion 3D cell culture system. It consists of two plates, the cell culture plate and the perfusion channel plate. In the cell culture plate, the four culture wells are designed to have the same size with the wells in the twelve well 2D culture plate. The edge of each well is partly cut to guide overflown medium to an inclined and open drain channel. The two inlets are connected to the sudden expansion reservoirs to trap incoming air bubbles before they enter into the perfusion system. In the perfusion channel plate, the linear concentration gradient generator is consisted of four branch microchannels with different lengths. The meandered microchannel mixers are connected to the outlets of the linear concentration gradient generator to mix the laminated media introduced from two facing branch channels efficiently.

Firstly, the air bubble trapping reservoir was designed. Air bubbles may exist in the medium or nutrients or can be generated in the various parts of the perfusion system. When the air bubbles adhere on the surface of the microchannels, the stability of the perfusion flow can be disturbed seriously because they change flow resistance. Specifically, it becomes more crucial when they block the microchannels because the perfusion flow into the microchannel is drastically reduced [24]. As a result, the cell growth is affected, and cell viability is also decreased [21, 22]. Previous work suggested bubble trap to remove air bubbles by buoyancy in a cylindrical chamber. The ceiling of the chamber was connected to bypass channel by using bypass tubing and a filter was inserted into the outlet of the bypass channel. Through the opened filter, there are concerns of biological contamination and spill of liquid.

To end these concern, an integrated chamber was considered to trap the air bubbles before entering into the perfusion system. The chamber connected to the inlet was shaped with a sudden expansion reservoir. When the air bubbles in the medium or nutrients flow through the inlet and move into the reservoir, they rise due to buoyancy resulting from the difference in density. The rising velocity of an air bubble can be calculated from the balance between buoyancy force and drag force acting on the air bubble. The perfusion velocity is also obtained by dividing the perfusion flow rate with the cross-sectional area of the channel. The rising velocity, $v_{\text {rising }}$ and the perfusion velocity, $v_{\text {flow }}$ are expressed in (1) and (2), respectively [31].

$$
v_{\text {rising }}=\frac{g \Delta \rho d_{\text {bubble }}}{18 \mu}
$$




$$
v_{\text {flow }}=\frac{Q}{A}
$$

where $g$ is the acceleration of gravity; $\Delta \rho$ is the density difference between the air bubble and the surrounding liquid; $d_{\text {bubble }}$ is the diameter of the air bubble; $Q$ is the perfusion flow rate, and $A$ is the cross-sectional area of the microchannel. To trap the air bubbles efficiently, they must float before entering to the linear concentration gradient generator. Thus, the air bubbles are trapped by buoyancy when the rising velocity is larger than the perfusion velocity. From the condition to float the air bubbles, the minimum diameter of the spherical air bubbles for trapping can be derived in (3). For a maximum perfusion flow rate of $200 \mu 1 / \mathrm{min}$ used in the experiment and the cross-sectional area of width and depth of 0.5 and $2.0 \mathrm{~mm}$, the minimum diameter was calculated to be $8 \mu \mathrm{m}$. The air bubbles with a diameter of below $8 \mu \mathrm{m}$ hardly clog the microchannels with a cross-sectional area of 1 $\mathrm{mm}^{2}$. Additionally, the bubble size could be decreased when the perfusion flow rate is much lower than 200 $\mu \mathrm{l} / \mathrm{min}$.

$$
d_{\text {bubble }}>\frac{18 \mu Q}{g \Delta \rho A}
$$

To determine the trapping capacity of the air bubbles, the dynamic behaviour of the infused air bubbles was simulated with commercial programs (CFD-GEOM and CFD-ACE+). The 2-dimensional numerical domain of the air bubble trapping reservoir was projected, and the width and height of the reservoir were 10 and $6 \mathrm{~mm}$, respectively. Initially, the reservoir was filled with water. Air bubbles with a diameter of $3 \mathrm{~mm}$ were set with a distance of $2 \mathrm{~mm}$ and infused successively with a perfusion flow rate of $200 \mu \mathrm{l} / \mathrm{min}$. A two-phase flow was analyzed with Flow and Free Surface (VOF) module in CFD-ACE+. To simulate the behavior of trapping air bubbles, gravity and surface tension were activated. The transient analysis showed that the air bubbles floated into the reservoir and collected as much as approximately $55.7 \mathrm{~mm}^{2}$, and the volume of the reservoir was $60 \mathrm{~mm}^{2}$ shown in Figure 2. This implies that the trapping capacity of air bubbles can be obtained from the volume of the sudden expansion reservoir. The reservoir was elliptically shaped with a major length, minor length and height of 10, 5.5 and $6 \mathrm{~mm}$, respectively. The trapping capacity of the reservoir was $260 \mu \mathrm{l}$.

(a)

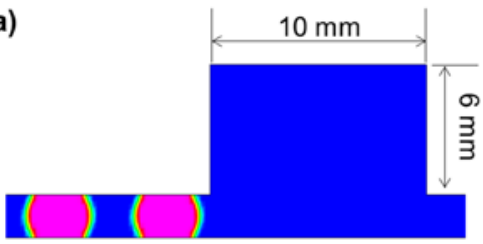

(c)

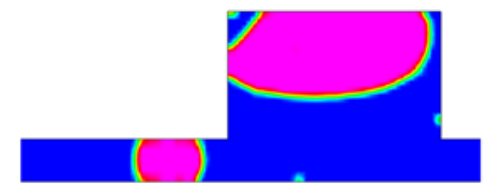

(b)

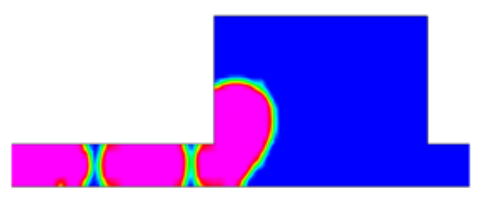

(d)

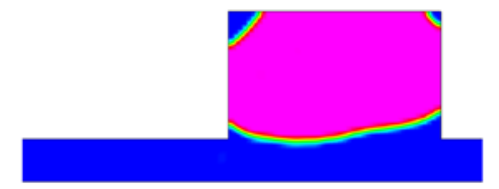

Figure 2. CFD analysis of behaviour of air bubbles in the air bubble trapping reservoir. (a) Set of air bubbles, (b) infusion with a perfusion flow rate of $200 \mu \mathrm{l} / \mathrm{min}$., (c) (d) collected air bubbles in the reservoir. 
Secondly, the linear concentration gradient generator was also integrated for high-throughput analysis. The mass concentration can be obtained simply by mixing two different liquids with a volume ratio. In the perfusion system, the volume can be replaced with the volumetric flow rate to control the concentration. Figure 3 shows four different linear concentration gradient generator. The main microchannel is bifurcated into four branch microchannels.

To distribute the volumetric perfusion flow rates linearly, the flow resistance of the microchannels was considered. From Poiseuille flow, the flow resistance can be obtained by dividing the pressure drop with the perfusion flow rate, analogous to Ohm's law in electricity, and it is expressed in (4).

$$
R_{f}=\frac{\Delta P}{Q}=\frac{128 \mu L}{\pi D_{h}{ }^{4}}
$$

where $R_{f}$ is the flow resistance; $\Delta P$ is the pressure drop; $Q$ is the perfusion flow rate; $D_{h}$ is the hydraulic diameter; $\mu$ is the dynamic viscosity of a fluid, and $L$ is the channel length. The flow resistance is proportional to the length and reversely proportional to the fourth power of the hydraulic diameter of the branch microchannel. Thus, in the design of the linear concentration gradient generator, the length of the branch microchannel was varied to control the volumetric perfusion flow rate instead of the hydraulic diameter because a small variation in the diameter resulted from fabrication accuracy affects a large change in the flow resistance.

In the design, four different concentrations of $40 \%, 30 \%, 20 \%$ and $10 \%$ were considered for the four perfusion culture wells, which were the linear concentration gradient. When a drug and a medium are introduced through two the inlets, the drug is divided into the four branch microchannels with a ratio of $40 \%, 30 \%, 20 \%$ and $10 \%$ of the perfusion flow rate and the medium with a ratio of $10 \%, 20 \%, 30 \%$ and $40 \%$. Then, they meet with the ratios of the volumetric flow rates of 4:1, 3:2, 2:3 and 1:4, respectively. To divide the inlet flow rate with the linear ratio of $40 \%, 30 \%, 20 \%$ and $10 \%$, the flow resistance network was analyzed. The flow resistances of the four branch microchannels and the connecting channels between each branch channel were calculated. Each flow resistance can be expressed in (5) by using (4) in the flow resistance network.

$$
R_{f, n}=\frac{Q_{n+1}\left(R_{f, g a p}+R_{f, n+1}\right)+\sum_{i=n+2} Q_{i} R_{f, g a p}}{Q_{n}}
$$

where $n$ is the branch microchannel number, and $R_{f, g a p}$ is the flow resistance of the connecting channel.

Figure 3 shows the linear concentration gradient generator. Each branch microchannel was spaced with a distance of $26 \mathrm{~mm}$ to arrange the wells with a diameter of $22.5 \mathrm{~mm}$ above the channel. The lengths of the branch microchannels were calculated to be 71, 41, 21 and $15 \mathrm{~mm}$, respectively. Moreover, to minimize minor losses and to secure size compactness, the branch microchannels were machined to be bent smoothly.

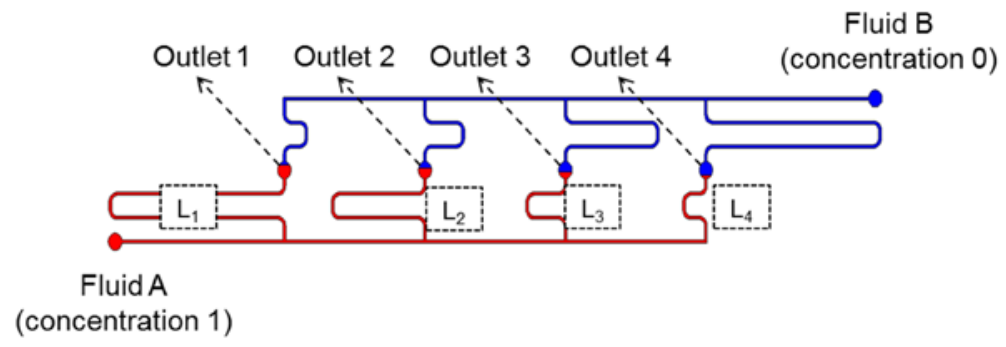

Figure 3. Design of the linear concentration gradient generator. 
A numerical analysis of the volumetric flow rates in the branch microchannels was conducted which proved that a linear concentration gradient of $40 \%, 30 \%, 20 \%$ and $10 \%$ within an error of less than $2 \%$ could be generated. Also, the color ratio of blue and red at the outlet indicates the ratio of the flow rates to obtain the linear gradient of concentration.

Thirdly, the microchannel mixer was designed. When the chemical and medium are ejected from the four outlets of linear concentration gradient generator and are introduced through microchannel, they form a laminar flow because the viscous effect is dominant in a low Reynolds number as a result of a small hydraulic diameter of the microchannel. Thus, they are required to be mixed well to obtain uniform concentration of their mixture in the perfusion culture wells. However, due to the difficulty of using turbulent mixing as well as ultrasonic or magnetic stirring in a laminar flow, the mixing is governed mainly by diffusion through the interface of two laminated fluids in the microchannel [11]. The enough length of the microchannel to complete the mixing of the two laminated fluids can be calculated by multiplying the diffusion time, $\tau$ and the fluid velocity as expressed in (6). The velocity of the fluid flow is also obtained by dividing the perfusion flow rate with the cross-sectional area [32].

$$
L_{\text {mixing }}=u \tau=u \frac{w^{2}}{2 D_{h}}=\frac{Q}{2 h_{\text {ratio }} \cdot D_{h}}
$$

where $u$ is the perfusion velocity; $\tau$ is the mixing time; $w$ is the microchannel width; $h_{\text {ratio }}$ is the aspect ratio of the microchannel defined by the ratio of the width and height, and $D_{h}$ is the hydraulic diameter.

Additionally, to improve the mixing efficiency and size compactness, the meandered microchannel mixer was compared with a straight microchannel mixer. The numerical analysis of mixing was conducted along the microchannel. In the analysis, two fluids of fluorescent Rhodamine 110 and water were considered, and the diffusion coefficient was $0.89 \times 10^{-9} \mathrm{~m}^{2} / \mathrm{s}$ at room temperature. The perfusion flow rate was $200 \mu 1 / \mathrm{min}$ through each inlet, and then, the flow rate entering each microchannel mixer was $100 \mu \mathrm{l} / \mathrm{min}$. The width and depth of the microchannel mixer was 0.5 and $2 \mathrm{~mm}$, respectively. The theoretical length to complete the diffusion mixing was calculated to be $117 \mathrm{~mm}$ from (6). Also, the Flow module and Chemical/Mixing module of the commercial program (CFD-ACE+) was used to analyze the mixing efficiency. Rhodamine 110 with a concentration of 1.0 was introduced with a flow rate of $50 \mu \mathrm{l} / \mathrm{min}$ and pure water with a concentration of 0.0 was also introduced with the same flow rate. The total perfusion flow rate was $100 \mu \mathrm{l} / \mathrm{min}$ in the microchannel mixer. The mixing ratio was evaluated by calculating the coefficient of variation (hereafter, C.V.) of the concentration of the Rhodamine 110 on the cross-sectional plane along the microchannel mixer. The C.V. is defined in (7).

$$
C . V .=\frac{\text { Standard deviation of concentration }}{\text { Mean of concentration }} \times 100(\%)
$$

Figure 4 compares the mixing efficiency between the straight microchannel mixer and the meandered microchannel mixer. To determine the uniform mixing on the cross-sectional plane, the mixing was evaluated when the C.V. was less than 5\%. In the straight microchannel mixer, a C.V. of $5 \%$ was obtained with a length of $120 \mathrm{~mm}$, which shows good agreement with the theoretical length of $117 \mathrm{~mm}$. In the meandered microchannel mixer, the mixing length was $60 \mathrm{~mm}$. It was reasoned that the convection term of transportation in each bent corner caused enhanced mixing. Finally, the linear concentration gradient generator was also simulated by connecting the branch microchannels and the meandered microchannel mixers with a length of $60 \mathrm{~mm}$. Figure 5 shows the linear concentration gradient generator with ratios of $39.7 \%, 29.9 \%, 20.0 \%$ and $10.4 \%$. The error was evaluated to be less than $4 \%$ compared with the design values. 


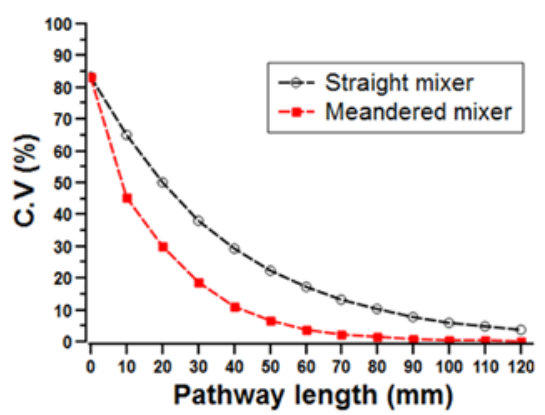

Figure 4. Coefficient of variation of the concentration in the microchannel mixers.

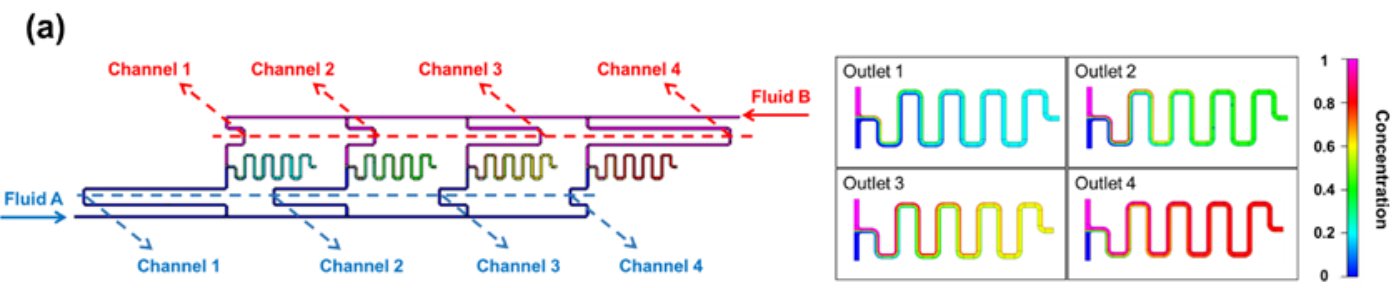

(b)

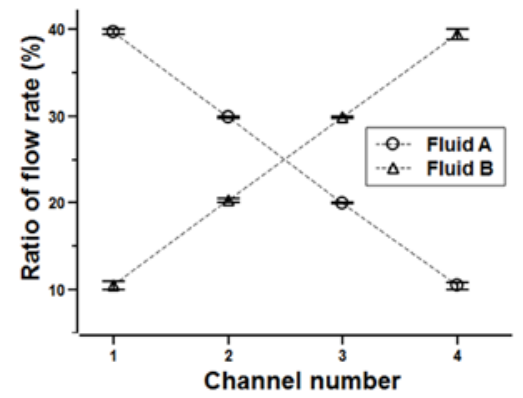

(c)

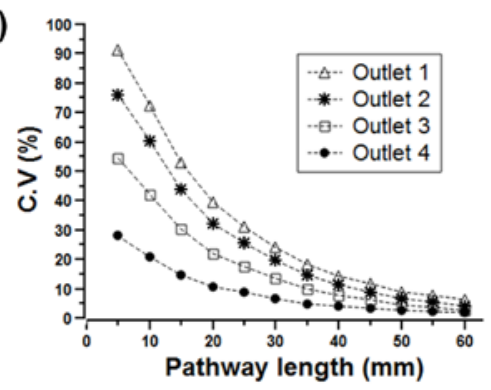

Figure 5. Numerical analysis of the perfusion microchannel. (a) Schematic of the checking points for performance evaluations, (b) linear distribution of the flow rates, (c) coefficient of variation of the concentration at the four outlets.

\section{Fabrication of the microfluidic perfusion system for 3D cell culture}

The microfluidic perfusion 3D cell culture well plate was fabricated by bonding two plates of biocompatible and transparent PMMA. Two inlets, air bubble trap reservoirs, perfusion culture wells, and a drain conduit were integrated into the upper cell culture plate. The open drain channel was machined with an inclined angle of $7^{\circ}$ to guide the overflown media from the perfusion culture wells by gravity. The four cell culture wells were fabricated with the same size as those in a 2D twelve well plate for user familiarity and convenience.

The 3D cell culture models can be divided into scaffold free system for spheroid culture and scaffold-based system for tissue culture representatively [33]. In this work, the scaffold (Alvetex, Reinnervate) was used for the 3D cell culture. Thus, the size and shape of the wells were machined to fit the scaffolds. At the center of the bottom of each well, a hole was bored to supply media continuously. The linear concentration gradient generator connected with the meandered microchannel mixers was machined in the bottom perfusion channel plate. To bond the well plate and the channel plate, a transparent and UV curable adhesive (MP-4102, CALOß) was coated onto the bottom of the upper well plate to minimize filling the microchannels with the adhesive during bonding. The coating thickness was about $50 \mu \mathrm{m}$. 


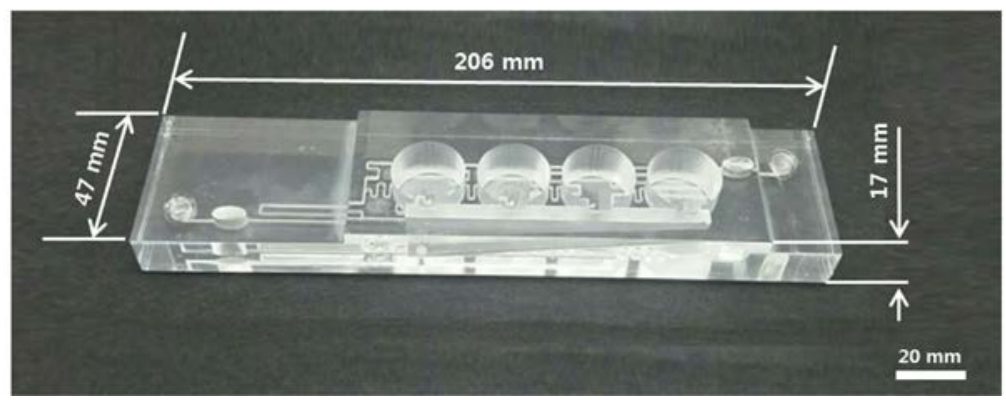

Figure 6. microfluidic perfusion 3D cell culture system fabricated with biocompatible PMMA.

After the outlets of the microchannel mixers were aligned with the holes of the culture wells and the two plates were bonded physically, UV was irradiated onto the edges for 5 seconds at 9 Watt for initial bonding and then, onto the whole surface for 30 minutes at 9 Watt. Figure 6 shows the fabricated microfluidic perfusion 3D cell culture system. To evaluate the bonding quality, the blockage of the microchannels due to the adhesive filling and the leakage of the unbound parts were examined. When water was introduced with a large flow rate of $500 \mu \mathrm{l} / \mathrm{min}$, any local blockage was not observed in the microchannels. Then, the outlets of the perfusion cell culture system were closed and no leakage was observed in the system.

\section{Performance evaluation of the microfluidic perfusion 3D cell culture system}

For the experimental evaluation, the perfusion flow rate entering the microfluidic perfusion 3D cell culture system was controlled by a syringe pump (PHD 2000, Harvard). First, the trapping capacity of the air bubble trap reservoir was tested. Initially, the microfluidic perfusion 3D cell culture system was filled with blue colored water for visualization. Then, air of $10 \mathrm{ml}$ was injected by using the syringe with a flow rate of $50 \mu \mathrm{l} / \mathrm{min}$. The trapping of the air bubbles in the reservoir was monitored over time. Figure 7 shows that the air bubbles in the reservoir were trapped successfully due to buoyancy. The trapping capacity of the reservoir was measured until the air bubbles started to enter into the microchannel. It was measured to be $268 \mu \mathrm{l}$, which agrees with the designed capacity. The small discrepancy was caused by the extra volume as a result of the meniscus of the air bubble.

Second, the linear concentration gradient generator was examined. Water mixed with Rhodamine 110 was injected into one inlet while only pure water was injected into the other inlet. To obtain a reference fluorescence intensity versus concentration, Rhodamine 110 with a controlled concentration ranging from 0.1 to 10 was prepared, and the fluorescence intensity of Rhodamine 110 was measured for the different concentrations with a microplate reader (FLUOstar, OPTIMA). The calibration curve was fitted with an $\mathrm{R}^{2}$ of 0.9820 shown in Figure 8.
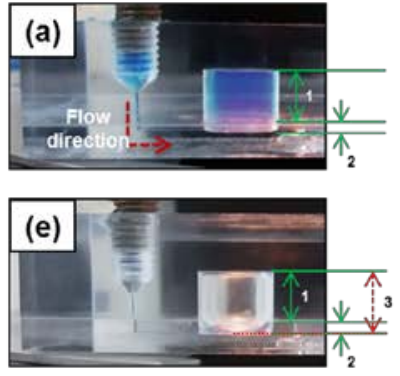
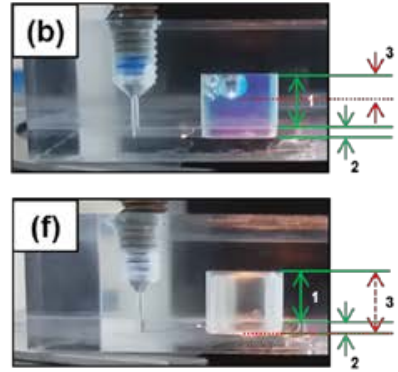
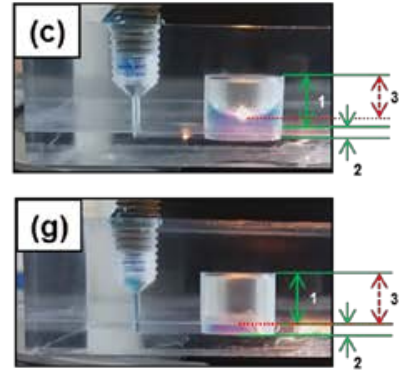

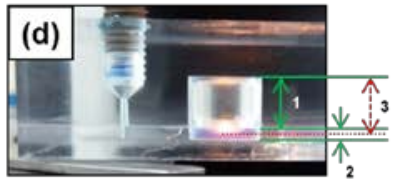

1. Bubble trap reservoir height

2. Channel height

3. Trapped bubble size

Figure 7. Visualization of air bubble trapping. (a) Air bubble trapping reservoir filled with water, (b) (f) during the infusion of air, (g) infusion of water after the reservoir was filled with air. 


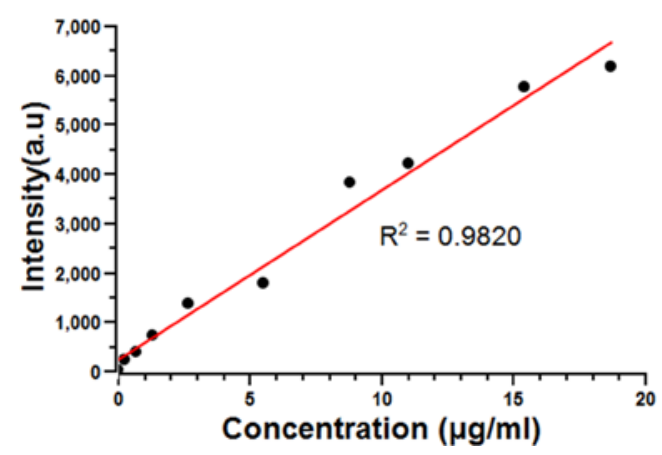

Figure 8. Calibration of Rhodamine 110 concentration

The inlet flow rate of the water mixed with the Rhodamine 110 and the pure water was $80 \mu \mathrm{l} / \mathrm{min}$. for both. The drained water was collected every 30 minutes. The syringes were refilled every 6 hours and three times. During the refilling process of the water, the air bubbles moving into the perfusion plate were observed. The fluorescence intensity of the drained samples was also measured. Figure 9(a) shows that the linear concentration gradient of $40 \%, 30 \%, 20 \%$ and $10 \%$ in the four perfusion wells was generated with an error of $9.7 \%$. The C.V. was calculated to be $1.9 \%$ on average by taking samples several times shown in Figure 9(a). Moreover, the performance of the linear concentration gradient generator was tested without the air bubble trapping reservoirs. As soon as air bubbles were introduced into the branch microchannels, the flow entering the linear concentration gradient generator was seriously disturbed and detoured into the rest of the branch microchannels. As a result, the linear concentration gradient was no longer consistently maintained shown in Figure 9(b).

(a)

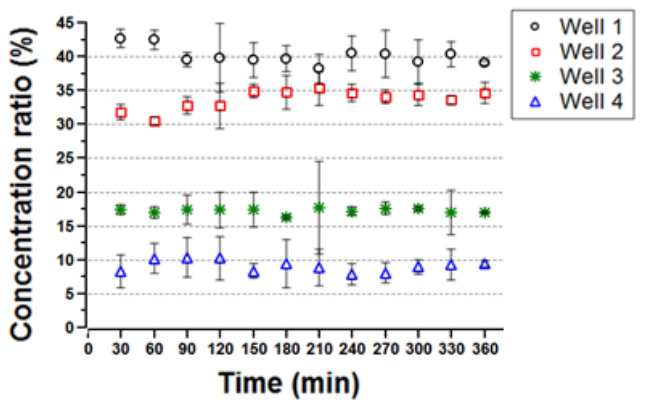

(b)

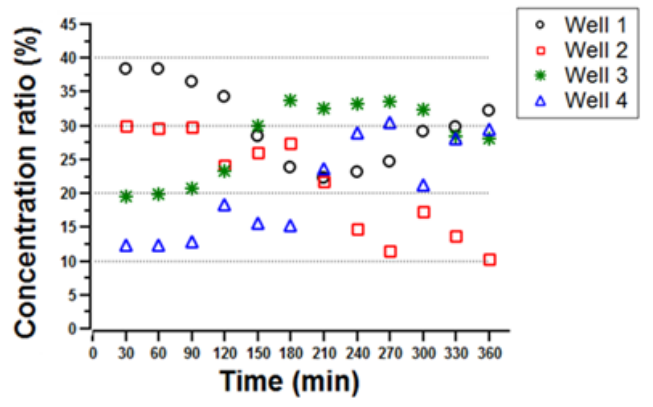

Figure 9. Performance of the linear concentration gradient of Rhodamine 110. (a) Perfusion test with the air bubble trapping reservoir, (b) without the reservoir.

\section{Application of the microfluidic perfusion 3D cell culture system to a cell cytotoxicity test}

The microfluidic perfusion 3D cell culture plate was used for a cytotoxicity test of Ewing's sarcoma cells, A673 (ATCC ${ }^{\circledR}$ CRL-1598 ${ }^{\mathrm{TM}}$ ), which are rare tumor cells and mostly expressed in the bones or tissues of children. It is not clearly known what causes Ewing's sarcoma. However, experimental reports have shown that the concentration of hydrogen peroxide, $\mathrm{H}_{2} \mathrm{O}_{2}$, has a key role in cancer development [34]. Thus, the toxicity test of the cells used different concentrations of $\mathrm{H}_{2} \mathrm{O}_{2}$ as a cytotoxic drug. For the perfusion 3D cell culture, the scaffolds were inserted into the four perfusion wells, and the Ewing's sarcoma cells were placed onto the scaffolds. Then, to mimic an in vivo environment, the media were warmed in a water bath, and the temperature was maintained 36 ${ }^{\circ} \mathrm{C}$. Moreover, the 3D perfusion cell culture plate was installed in a warm chamber to acclimate it to a temperature of $36^{\circ} \mathrm{C}$. By using a peristaltic pump (MNI PULS 3, Glison ${ }^{\circledR}$ ), the nutrient medium and a mixture of the medium and $\mathrm{H}_{2} \mathrm{O}_{2}$ were introduced into the two inlets, respectively, and the perfusion flow rate was $40 \mu 1 / \mathrm{min}$ for each. Specifically, the perfusion flow rate was selected by considering the designed linear concentration gradient 
generator in the four culture wells and the previous work using a perfusion cell culture with a perfusion flow rate ranging from 0.1 to $1 \mathrm{ml} / \mathrm{min}$. [35].

The perfusion cytotoxicity was assayed for 4 hours. During this time, $100 \mu$ of drained media were collected every 30 minutes and mixed with $10 \mu$ of propidium iodide (PI). Then, the fluorescence intensity was analyzed. The excitation and emission wavelengths were 540 and $620 \mathrm{~nm}$, respectively. The gain value was set as 1800 . The intensity of the PI relates the degree of cell death. The degree of cell death was measured for the four different concentrations of $\mathrm{H}_{2} \mathrm{O}_{2}$. Figure 10 shows that cell death increased continuously until up to 240 minutes, while the specific threshold concentration of the cell death was not found. Moreover, more cells were killed at the higher concentration. A higher PI signal was observed at a higher concentration of $\mathrm{H}_{2} \mathrm{O}_{2}$, and there were less cell proteins on the scaffold. To conduct long term culture assays using the developed perfusion cell culture system, the microfluidic perfusion 3D cell culture plate needs to increase its capacity for air bubble trapping, after which, it needs to be compared with a static 2D cell culture of the same cells.

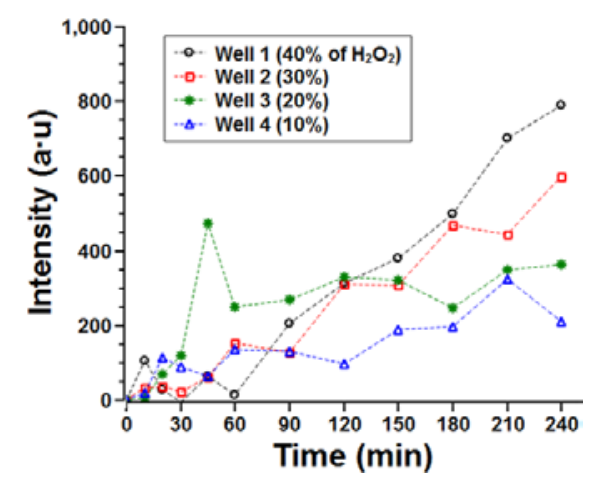

Figure 10. Result of the PI stained DNAs from Ewing’s sarcoma cells.

\section{Conclusions}

A microfluidic perfusion 3D cell culture system consisting of air bubble trapping reservoirs, gradient generators to provide a linear concentration and meandered micromixers for nutrients or drugs was developed in this study to examine cell cultures with a closer relevance to in vivo microenvironments. The trapping capacity of the sudden expansion reservoir for air bubbles was determined by transient numerical analysis and visualization. Both methods showed that the incoming air bubbles float upward in the sudden expansion trapping reservoir due to the buoyancy force and gather until the reservoir is full. The maximum trapping capacity of the reservoir was determined to be the same volume as the sudden expansion reservoir. Additionally, the performance of the linear concentration gradient generator fluidically connected with the meandered microchannel mixer was examined by measuring the fluorescence intensity of Rhodamine 110 in the perfusion cell culture wells. The linear concentration in the wells had a ratio of $40 \%+1 \%, 30 \%+4 \%, 20 \%-3 \%$ and $10 \%-1 \%$. In addition, the linear gradient concentration in the wells was stably maintained for a long-term cell culture with the air bubble trapping reservoir, while the concentration in the wells without it was seriously disturbed. In this fabrication of the microfluidic perfusion 3D cell culture plate, two biocompatible and transparent PMMA plates were bonded with a UV curable adhesive. However, the bonding can be improved by using thermal compression of the two plates for mass-manufacturing. The suggested microfluidic perfusion 3D cell culture plate is potentially applicable to high throughput screening of drugs, nutrients, and growth factors. For future works, its comparison with a static 2D cell culture well plate should be done for a specific targeted cell.

\section{Acknowledgments}

This research was financially supported by the National Research Foundation of Korea (NRF) grant funded by the Korea government (MOE) (No. NRF-2014R1A1A2057489) and (No. NRF-2017R1A2B2006264). 


\section{References}

[1] Back C Y and Min J H 2015 Optional cell patterning techniques J. KSME 5511 46-52

[2] Sanyal S and Kosovsky M 2015 Materials and assay systems used for 3D cell Culture The Cell Culture Dish

[3] Baker B M and Chen C S 2012 Deconstructing the third dimension - How 3D culture microenvironments alter cellular cues J. Cell Sci. 125 3015-24

[4] Caicedo-Carvajal C E, Liu Q, Remache Y, Goy A and Suh K S 2011 Cancer tissue engineering: A novel 3D polystyrene scaffold for In Vitro isolation and amplification of lymphoma cancer cells from heterogeneous cell mixtures $2011 \mathrm{~J}$. Tissue Eng.

[5] Tung Y C, Hsiao A Y, Allen S G, Torisawa Y, Ho M and Takayama S 2011 High-throughput 3D spheroid culture and drug testing using a 384 hanging drop array Anal. 136 473-8

[6] Li X J, Valadez A V, Zuo P and Nie Z 2012 Microfluidic 3D cell culture: potential application for tissuebased bioassays Bioanal. 412 1509-25

[7] Ziolkowska K, Hofman I, Dybko A, Brzozka Z 2012 Integrated passive bubble trap for long-term cell culture microfluidic systems MicroTas 938-40

[8] Kim L, Toh Y C, Voldman J and Yu H 2007 A practical guide to microfluidic perfusion culture of adherent mammalian cells Lab Chip 7 681-97

[9] Caicedo-Carvajal C E and Liu Q 2012 Using a novel 3D perfusion bioreactor to culture $\beta$-actin-RFP reporter osteosarcoma Biowire Spring 25-8

[10] Tian K, Zhong W, Zhang Y, Yin B, Zhang W and Liu H 2015 Microfluidics-based optimization of neuroleukin-mediated regulation of articular chondrocyte proliferation Molecular Medicine Reports 67-74 [11] Walker G M, Riviere N M, Rouse J and O’Neill A T 2006 A linear dilution microfluidic device for cytotoxicity assays Lab. Chip 7 226-32

[12] Yang C C, Wen R C, Shen C R and Yao D J 2015 Using a microfluidic gradient generator to characterize BG-11 medium for the growth of cyanobacteria synechococcus elongates PCC7942 Micromach. 6 1755-67

[13] Toh Y C, Lim T C, Tai D, Xiao G, Noort D and Yu H 2009 A microfluidic 3D hepatocyte chip for drug toxicity testing Lab. Chip. 9 2026-35

[14] Cooksey G A, Sip C G and Folch A 2009 A multi-purpose microfluidic perfusion system with combinatorial choice of inputs, mixtures, gradient patterns, and flow rates Lab. Chip. 93 417-26 
[15] Jeon N L, Baskaran H, Dertinger S K W, Whitesides G M, Water L V D and Toner M 2002 Neutrophil chemotaxis in linear and complex gradients of interleukin-8 formed in a microfabricated device Nat. Biotech. 20 826-30

[16] Fujii S I, Uematsu M, Yabuki S, Abo M, Yoshimura E and Sato K 2006 Microbioassay system for an anti-cancer agent test using animal cells on a microfluidic gradient mixer Anal. Sci. 22 87-90

[17] Selimovic S, Sim W Y, Kim S B, Jang Y H, Lee W G, Khabiry M, Bae H, Jambovane S, Hong J W and Khademhosseini 2011 Generating non-linear concentration gradient in microfluidic devices for cell studies Anal. Chem. 836 2020-8

[18] Abhyankar V V, Lokuta M A, Huttenlocher A and Beebe D J 2006 Characterization of a membranebased gradient generator for use in cell-signaling studies Lab. Chip. 6 389-93

[19] Dertinger S K W, Chiu D T, Jeon N L and Whitesides G M 2001 Generation of gradients having complex shapes using microfluidic networks Anal. Chem. 73 1240-6

[20] Thompson D M, King K R, Wieder K J, Toner M, Yarmush M L and Jayaraman A 2004 Dynamic gene expression profiling using a microfabricated living cell array Anal. Chem. 76 4098-103

[21] Zheng W, Wang Z, Zhang W and Jiang X 2010 A simple PDMS-based microfluidic channel design that removes bubbles for long-term on-chip culture of mammalian cells Lab Chip 2906-10

[22] Kang E, Lee D H, Kim C B, Yoo S J and Lee S H 2010 A hemispherical microfluidic channel for the trapping and passive dissipation of microbubbles J. Micromech. Microeng. 204

[23] Zhang Y S, Chae S K, Polini A, Dokmeci M R and Khademhosseini A 2014 A highly efficient bubble trap for continuous removal of gas bubbles from microfluidic devices MicroTas 730-2

[24] Stucki J D and Guenat O T 2015 A microfluidic bubble trap and oscillator Lab Chip 4393-7

[25] Sung J H and Shuler M L 2009 Prevention of air bubble formation in a microfluidic perfusion cell culture system using a microscale bubble trap Biomed. Microdev. 11 731-8

[26] Lochovsky C, Yasotharan S and Gunther A 2010 Bubbles no more: trapping and removal of gas bubbles in single-layer elastomeric devices MicroTas 1805-7

[27] Kim C, Lee K, Kim J H, Shin K S, Lee K J, Kim T S and Kang J Y 2008 A serial dilution microfluidic device using a ladder network generating logarithmic or linear concentrations Lab. Chip. 8 473-9

[28] Lee P J, Hung P J and Lee L P 2007 An artificial liver sinusoid with a microfluidic endothelial-like barrier for primary hepatocyte culture Biotech. Bioeng. 975

[29] Ong S M, Zhang C, Toh Y C, Kim S H, Foo H L, Tan C H, Noort D, Park S and Yu H 2008 A gel-free 3D microfluidic cell culture system Biomat.29 3237-44 
[30] Beskardes I G, Hayden R S, Glettig D L, Kaplan D L and Gumusderelioglu M 2017 Bone tissue engineering with scaffold-supported perfusion co-cultures of human stem cell-derived osteoblasts and cell line-derived osteoclasts Proc. Biochem. 59 303-11

[31] Zheng L and Yapa P D 2000 Buoyant velocity of spherical and nonspherical bubbles/droplets $J$. Hydraulic Eng. 126 852-4

[32] Nguyen N T and Wereley S T 2006 Fundamentals and applications of microfluidics $2^{\text {nd }}$ edition Artech House 357-63

[33] Jenkins J, Dmitriev R I, Morten K, McDermott K W and Papkovsky D B 2015 Oxygen-sensing scaffolds for 3-dimensional cell and tissue culture Acta Biomat. 16 126-35

[34] Lazaro M L 2007 Dual role of hydrogen peroxide in cancer: possible relevance to cancer chemoprevention and therapy Cancer Letters 252 1-8.

[35] Cartmell S H, Porter B D, Garcia A J and Guldberg R E 2003 Effects of medium perfusion rate on cellseeded three-dimensional bone constructs in vitro Tissue Eng. 96 1197-203 